\title{
Diagnostic Efficiency of Multidetector Computed Tomography in the Evaluation of Clinically Equivocal Cases of Acute Appendicitis with Surgical Correlation
}

\author{
Jawaid Iqbal Dr. $^{1}$, Raza Sayani ${ }^{2}$, Misbah Tahir Dr ${ }^{1}$, Syed M. Mustahsan ${ }^{3}$ \\ 1. Radiology, Liaquat National Hospital and Medical College, Karachi, PAK 2. Department of Radiology, \\ The Aga Khan University, Karachi, PAK 3. Emergency Medicine, The Aga Khan University Hospital, \\ Karachi., Karachi, PAK
}

$\square$ Corresponding author: Raza Sayani, sayani_raza@yahoo.com

Disclosures can be found in Additional Information at the end of the article

\section{Abstract}

Acute appendicitis is one of the most frequent causes of lower abdominal pain and requires immediate surgical intervention. The diagnosis often poses a lot of challenge even to experienced surgeon. Those patients with equivocal symptoms may require different imaging modalities like radiography, contrast examination and ultrasound with limited utility. Multidetector computed tomography (MDCT) used in suspected acute appendicitis has, however, resulted in improved diagnostic accuracy and also reduction of negative surgeries.

\section{Objective}

We intend to determine the diagnostic efficiency of MDCT in clinically equivocal cases of acute appendicitis correlating it with surgical/histopathological findings.

\section{Materials and methods}

A group of 116 patients was included in this study. Spiral MDCT was performed in all these cases after administration of oral and intravenous contrast. All these patients underwent surgery and the CT findings were correlated with histopathology. Out of these 116 patients, 60 patients were male and 56 female. The age range was from three to seventy years and mean age was $28+1$ years.

\section{Results}

Received 02/13/2018

Review began 02/15/2018 Review ended 02/22/2018 Published 03/01/2018

\section{(c) Copyright 2018}

Iqbal et al. This is an open access article distributed under the terms of the Creative Commons Attribution License CC-BY 3.0., which permits unrestricted use, distribution, and reproduction in any medium, provided the original author and source are credited.
The results proved that MDCT had a sensitivity of $97.5 \%$, specificity of $97.0 \%$, and accuracy of 97.4\% for the diagnosis of appendicitis with one false positive and two false negative cases. The study showed $100 \%$ accuracy in diagnosing acute appendicitis in children. In 33 patients, an alternate cause was identified with CT. The alternate diagnosis made on CT findings was consistent with the final diagnosis in 27 (81.8\%) of 33 patients in whom there was no evidence of acute appendicitis. The clinical diagnosis disagreed with the CT diagnosis in six patients (18.18\%).

\section{Conclusion}

Our study verifies that MDCT plays an important role in evaluation and consequent management of equivocal cases of acute appendicitis. MDCT is also able to diagnose appendicitis or detect alternative diagnosis in pediatric population.

How to cite this article

Iqbal J, Sayani R, Tahir M, et al. (March 01, 2018) Diagnostic Efficiency of Multidetector Computed Tomography in the Evaluation of Clinically Equivocal Cases of Acute Appendicitis with Surgical Correlation. Cureus 10(3): e2249. DOI 10.7759/cureus.2249 
Categories: Pathology, Radiology, General Surgery

Keywords: acute appendicitis, diagnostic efficiency, computed tomography

\section{Introduction}

Acute appendicitis is one of the most frequent causes of lower abdominal and right lower quadrant pain. This requires immediate surgical intervention in the form of appendectomy, which is one of the most common surgical procedures. The diagnosis often poses a lot of challenge even to experienced surgeon as several other gynecological, gastrointestinal and genitourinary conditions can have similar presentation [1,2]. Those patients with equivocal symptoms and without acute appendicitis could not only be spared of unnecessary cost but also of the associated morbidity of surgery if proper imaging and appropriate evaluation were done beforehand. In contrast, the morbidity associated with missing or delaying the diagnosis has serious consequences including appendicular perforation, peritonitis, abscess formation, sepsis, and can even cause death [3,4].

The clinical diagnosis of acute appendicitis has around 20\% false-positive and a similar falsenegative error rate $[5,6]$. These patients will have equivocal clinical findings and/or laboratory test results thus making the diagnosis difficult [7-9]. To overcome this error rate different imaging modalities like radiography, contrast examination and ultrasound have been used with limited utility. Multidetector computed tomography (MDCT) used in suspected acute appendicitis has, however, resulted in improved diagnostic accuracy and also reduction of negative surgeries $[10,11]$.

The purpose of this study was to determine the diagnostic efficiency of non-focused contrastenhanced spiral CT abdomen to confirm or exclude appendicitis in patients who presented with equivocal signs and symptoms of appendicitis.

\section{Materials And Methods}

This was a comparative cross-sectional study performed at Departments of Radiology, Surgery and Pathology for a period of eight months. The sampling technique used was purposive with patients presenting either to the emergency department or outpatients' clinics with periumbilical and/or right lower quadrant pain.

Patients of all ages presenting with periumbilical and/or right lower quadrant pain with pyrexia and leucocytosis with initial ultrasound examination yielding results inconsistent with the clinical presentation were included.

Patients with prior appendectomy, acute appendicitis confirmed on ultrasound, pregnant females, those who were unable to receive either oral or intravenous contrast material, those who were discharged without surgery and patients with clinically unequivocal cases of appendicitis undergoing laparotomy without imaging were excluded.

Initially, 123 patients were included in the study. Out of these, seven patients were excluded from the study. Two patients had the history of appendectomy, IV contrast could not be administered to two patients having the history of chronic renal failure and were on dialysis and three patients were discharged without surgery. So, finally 116 patients were included in the study. Out of these, 60 patients were male and 56 female. The age ranged from three to seventy years and mean age was $28+1$ years.

All patients were restrained from taking anything orally. All CT scans were performed on 


\section{Cureus}

Toshiba Asteon, Multislice CT Scanner. Opacification of the gastrointestinal tract was achieved through the oral and rectal administration of $3 \%$ diatrizoate meglumine solution (Gastrografin). Scans were performed before and after administration of I/V contrast. The abdomen was helically scanned during a single breath-hold (pitch of 1.5:1, $120 \mathrm{kVp}, 240-320$ $\mathrm{mAs}$ ) from the dome of the diaphragm up to the symphysis pubis at an 8-mm collimation. A total of 1.5-2.0 ml of iodinated contrast was injected per kilogram of body weight, with doses based on age and weight with Medrad Vistron CT power injector system @2.0-3.0 ml/second. Informed consent was obtained from all patients. The image interpretation was done on the basis of primary and secondary diagnostic criteria for acute appendicitis. Acute appendicitis was labeled on CT scans if three or more of the following criteria were present.

Appreciation of a thickened/distended appendix which is $>6 \mathrm{~mm}$ whether it is with or without mural thickening and enhancement (Figure 1).

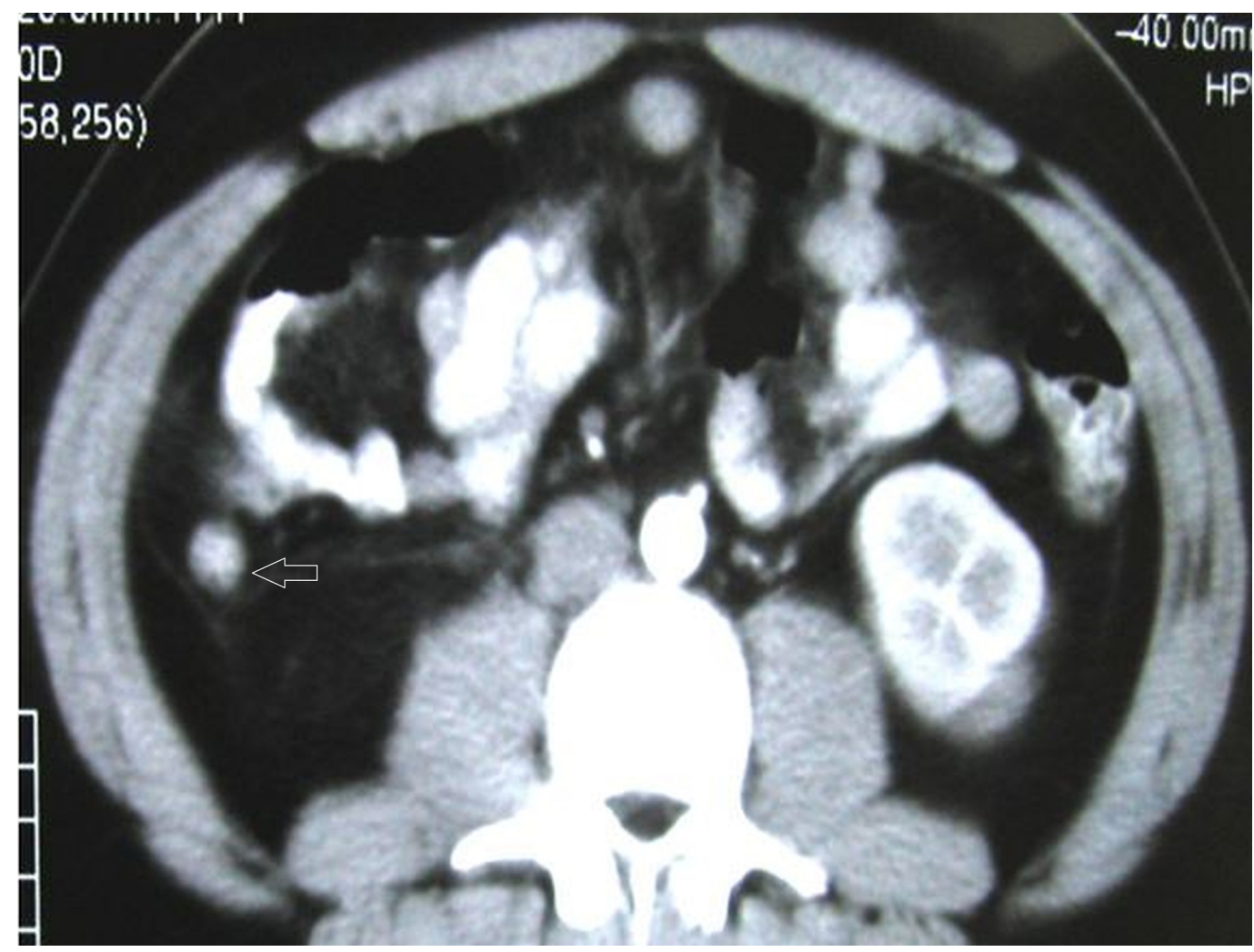

FIGURE 1: Acute appendicitis in a 21-year-old male. Axial contrast enhanced image showing dilated appendix measuring $11 \mathrm{~mm}$ (Arrow) in maximum thickness with enhancement of its walls.

Periappendiceal fat stranding which is represented by progressively increased haziness and linear areas of high attenuation in the periappendiceal fat (Figure 2). 


\section{Cureus}

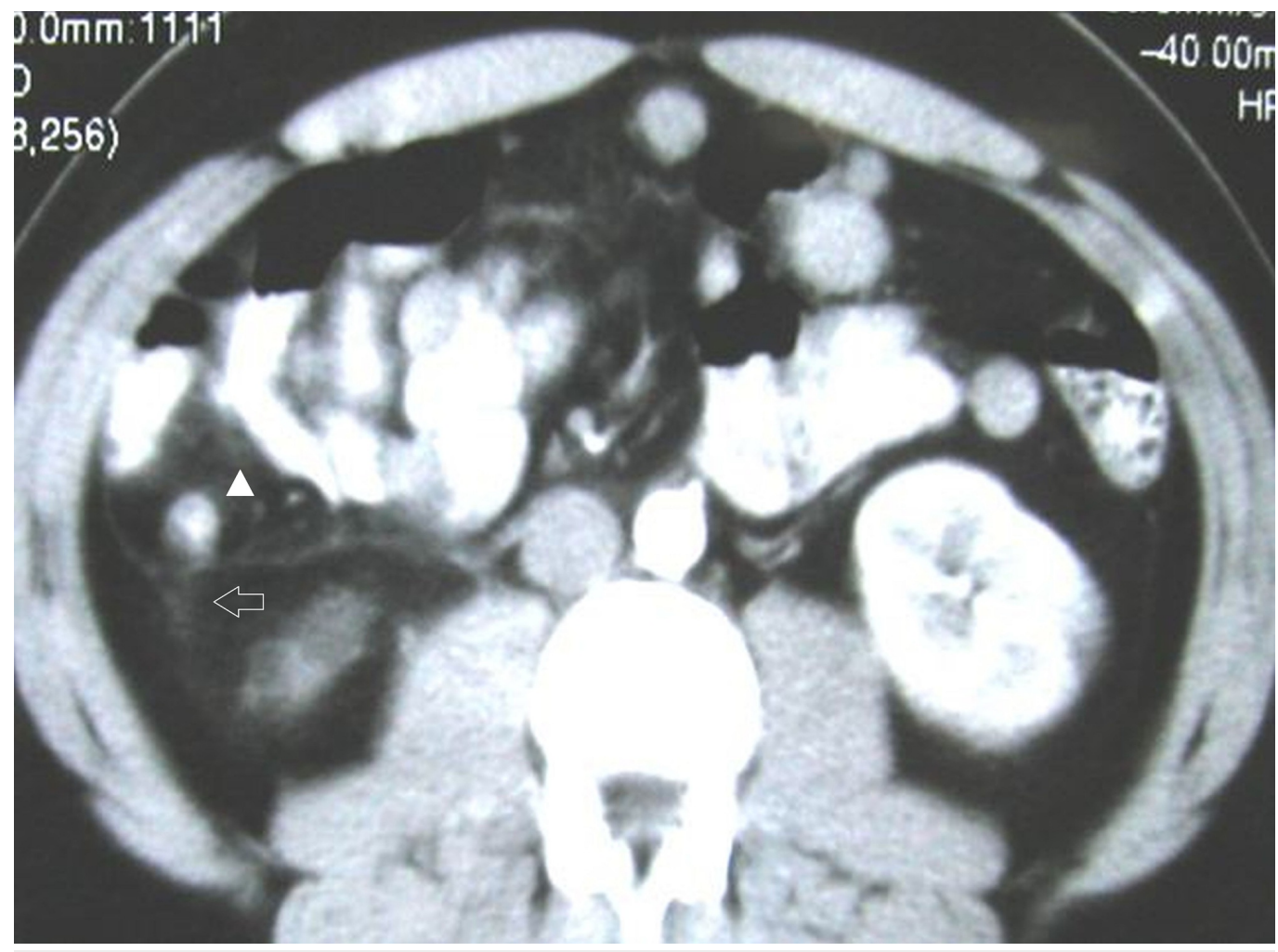

FIGURE 2: Acute appendicitis in a 41-year-old male. Axial contrast enhanced image showing periappendiceal fat stranding along with thickening of right Gerota's fascia (Arrow) and a dilated appendix (Arrowhead).

Secondary diagnostic criteria included the presence of appendicolith, periappendiceal abscess or phlegmon, mural thickening of the cecum, enlarged lymph nodes and extra-luminal air.

The scans were also evaluated for any other alternative diagnosis for the cause of symptoms. No case was lost to follow-up. In all selected 116 cases, the findings seen on the CT scans and surgical/pathological findings were collected and proforma was filled for each patient and they were compared with surgical and histopathology findings. Histopathological findings were taken as a gold standard for the diagnosis of appendicitis. Alternative diagnoses were evaluated in CT as well.

Data initially collected on proforma was then analyzed on SPSS Version 20. Different percentages and frequencies were obtained. Sensitivity, specificity, positive predictive value, negative predictive value, and accuracy were calculated on SPSS.

\section{Results}

The results of our study showed that all patients had histopathological diagnosis of either acute appendicitis with or without associated findings or any of the alternative diagnoses after the CT scan. Correct assessment for acute appendicitis was made in 113 of 116 scans (97\%). Incorrect assessment was made in three scans (3\%). Of the 113 scans correctly evaluated, 80 were true positive for acute appendicitis and 33 were true negative, i.e., alternative diagnosis.

Of the three CT scans proven to be incorrectly evaluated, acute appendicitis was falsely 
interpreted as positive in one patient and as negative in two patients. One case that turned out to be false positive was of a 60 -year-old female who had mucinous cystadenocarcinoma of appendix, a much rarer diagnosis. The cystic component of the mass was misinterpreted as the fluid-filled bowel not opacified with contrast.

In two false negative cases, the appendix was within the upper limits of the normal range of thickness. Marginal periappendiceal stranding was confirmed on retrospective analysis of the scans after the surgico-pathological outcome was known.

The diagnosis of acute appendicitis was made on the basis of presence or absence of major and minor criteria as discussed previously. In 80 patients with positive CT findings for acute appendicitis, the dilated non-opacified appendix ( $>6 \mathrm{~mm}$ in diameter) was visualized in 74 patients (92\%) as shown in Figure 1 and not visualized in six patients (7\%). In these six patients, an abscess in the RIF suggested a ruptured appendix.

In 33 patients with negative CT findings for acute appendicitis, normal appendix was seen in five patients (15\%) and not seen in 28 patients (85\%). In three out of the five cases in which normal appendix was seen, it was filled with contrast material, air was seen in its lumen in one case and in one patient no air or contrast was seen within the lumen but the appendix was of normal size.

Right lower quadrant fat stranding was a strong predictor of appendicitis. Among 80 patients with positive CT findings for acute appendicitis, moderate or severe stranding was identified in 53 patients (66\%) as shown in Figure 1. An appendicolith was detected at CT in $11(14 \%)$ patients, with an enlarged appendix in seven patients, and classical acute appendicitis, periappendiceal abscess/phlegmon were identified on CT scan in 14 cases (17\%). Extra-luminal air was seen in two patients (3\%) in case of perforation of the appendix along with a complex mass representing periappendiceal abscess or phlegmon. Abnormal cecal wall thickening was labeled on comparison with the normal wall thickness of ascending colon distal to the cecum and was seen in 23 patients (29\%). Isolated, enlarged lymph nodes $(>1.5 \mathrm{~cm})$ in the pelvis were seen in 16 patients (20\%) along with other findings for acute appendicitis especially in pediatric age group.

In 80 of 82 patients, acute appendicitis was diagnosed correctly on MDCT examination (sensitivity, 97.56\%). It was correctly excluded prospectively in 33 of 34 patients (specificity, 97\%). Overall accuracy for diagnosing acute appendicitis was $97.41 \%$. The positive and negative predictive values were $98.76 \%$ and $94.28 \%$ respectively seen.

In 33 (28.4\%) of 116 patients, alternative diagnosis was made with MDCT, including gynecologic abnormalities making up the largest proportion in 19 (57.5\%) patients. Out of the nine adnexal masses reported, the possibility of ectopic gestation was successfully raised in three cases. Colonic pathologies were seen in four (12.1\%), small bowel disease in six (18.1\%), renal infection with calculi in three (9\%) and acute cholecystitis was reported in one case (3\%). Of the true-negative cases, the alternative diagnosis based on CT findings matched with the final diagnosis in 27 (81.8\%) of 33 patients without acute appendicitis. The clinical diagnosis disagreed with CT diagnosis in six patients (18.18\%).

\section{Discussion}

Acute appendicitis may be a relatively straightforward diagnosis if it presents with typical signs and symptoms or when a distended, non-compressible thick-walled appendix is identified on ultrasound examination but the diagnosis may be more challenging when signs and symptoms are equivocal as in mild forms of appendicitis, where there may be minimal dilatation, less 
periappendiceal inflammation, small amount of retroperitoneal fat and in patients with significant pericaecal inflammation due to ruptured appendix [12,13].

MDCT has been shown to be highly precise in the diagnosis of acute appendicitis, though there is no consensus about which technique is ideal [14-16]. In our study, we scanned the entire abdomen and pelvis by using non-focused contrast-enhanced technique. This technique was also used by Balthazar et al. [17] and uses thin-section $(5 \mathrm{~mm})$ imaging through the pelvic region and thicker images in the upper portion of the abdomen. The principal reason for this approach is to pick other conditions which mimic appendicitis and have similar presentation $[13,18]$.

IV contrast usage is important to patients with suspected acute abdomen as it improves the ability to identify the inflamed appendix, and to establish alternative diagnoses [18]. IV contrast also allows better evaluation of wall enhancement of appendix, differentiation of pelvic blood vessels from a retro-cecal appendix [19]. Contrast material cost and reaction risks are definite disadvantages $[20,21]$, though no significant contrast reaction was seen in our study.

Studies with CT protocols without oral or per-rectal contrast agents have emphasized the time saved and patient discomfort $[13,15]$. Our patients did not complain of contrast intolerance or discomfort.

Rao et al. $[19,22]$ used limited CT of the lower abdomen after oral and per-rectal contrast material administration, the technique used by us differed in several aspects as they included all patients clinically suspected of having acute appendicitis, whereas we examined only those patients who presented with equivocal sign and symptoms of acute appendicitis. CT was not performed in clinically obvious cases of acute appendicitis as the referring surgeons did not expect an added advantage and feared increased complications.

Thin-section focused helical CT was used by Lane et al. for the detection of suspected acute appendicitis and reported detection rate of $100 \%$ [23]. We were able to identify abnormal appendix in 74 (92.5\%) of the 80 patients with acute appendicitis. We doubt the use of limited examination as a wide range of genitourinary and gastrointestinal conditions which mimic acute appendicitis and may be missed [24].

The major imaging findings of acute appendicitis at MDCT were based on thickened appendix measuring greater than $6 \mathrm{~mm}$ and periappendiceal inflammation [13,15]. Ours as well as the study by Rao et al. shows that periappendiceal inflammation is the most sensitive sign of acute appendicitis. An enlarged appendix of greater than $6 \mathrm{~mm}$ was $93 \%$ sensitive and $100 \%$ specific for acute appendicitis $[25,26]$. Additional findings on CT scans in acute appendicitis include cecal wall thickening, appendicoliths, and periappendiceal fluid collections [25].

One false positive case is our series was of a 60 -year-old female who was diagnosed mucinous cystadenocarcinoma of appendix on histopathological examination, a much rarer diagnosis. The cystic component of the mass was misinterpreted as the fluid-filled bowel not opacified with contrast. $90 \%$ of all appendiceal tumors are carcinoids but lymphomas and carcinomas can also involve the appendix [26,27]. Most are found incidentally at surgery for another procedure or for appendicitis. Metastases are exceedingly rare [28]. In two false negative cases, the diameter of appendix was at the higher limit of normal range of thickness and there was marginal periappendiceal fat stranding on retrospective review.

Our study showed $100 \%$ accuracy in diagnosing acute appendicitis in children. Studies have also shown decrease in the rate of perforation in children who underwent CT as compared to those who did not (15\% vs $23 \%$ ). Also, a significant decrease in the negative appendectomy rate was 
noted in children with suspected acute appendicitis who underwent CT as compared to those who did not (6\% vs $12 \%)[24,29]$.

In our study in 33 (28.4\%) of 116 patients, an alternative diagnosis was made with CT, including gynecologic abnormalities in 19 (57.5\%), colonic pathologies in four (12.1\%), small bowel disease in six (18.1\%), renal infection with calculi in three (9\%), and acute cholecystitis was reported in one case (3\%). This was in agreement with the study by Federle et al. and Rao et al. [23].

\section{Conclusions}

The study shows that non-focused contrast-enhanced multi-detector CT is highly accurate in diagnosing acute appendicitis or suggesting an alternative diagnosis in patients with equivocal signs and symptoms. Use of this rapid, non-operator dependent and highly accurate examination may decrease delays in appropriate medical or surgical therapy as well as unnecessary delayed observation.

\section{Additional Information \\ Disclosures}

Human subjects: Consent was obtained by all participants in this study. College of Physicians and Surgeons Pakistan issued approval RTMC-REC-20. Consent for scanning and research obtained. Animal subjects: All authors have confirmed that this study did not involve animal subjects or tissue. Conflicts of interest: In compliance with the ICMJE uniform disclosure form, all authors declare the following: Payment/services info: All authors have declared that no financial support was received from any organization for the submitted work. Financial relationships: All authors have declared that they have no financial relationships at present or within the previous three years with any organizations that might have an interest in the submitted work. Other relationships: All authors have declared that there are no other relationships or activities that could appear to have influenced the submitted work.

\section{References}

1. Yu J, Fulcher AS, Turner MA, et al.: Helical CT evaluation of acute right lower quadrant pain: part I, common mimics of appendicitis. Am J Roentgenol. 2005, 184:1136-1142. 10.2214/ajr.184.4.01841136

2. Bongard F, Landers DV, Lewis F: Differential diagnosis of appendicitis and pelvic inflammatory disease. Am J Surg. 1985, 150:90-96. 10.1016/0002-9610(85)90015-7

3. Pinto Leite N, Pereira JM, Cunha R, et al.: CT evaluation of appendicitis and its complications: imaging techniques and key diagnostic findings. Am J Roentgenol. 2005, 185:406-417. 10.2214/ajr.185.2.01850406

4. Eldar S, Nash E, Sabo E, et al.: Delay of surgery in acute appendicitis . Am J Surg. 1997, 173:194-198. 10.1016/S0002-9610(96)00011-6

5. Ramirez JM, Dues J: Practical scores to aid decision making in doubtful cases of appendicitis . Br J Surg. 1994, 81:680-683. 10.1002/bjs.1800810516

6. Lewis FR, Holcroft JW, Boey J, et al.: Appendicitis. A critical review of diagnosis and treatment in 1000 cases. Arch Surg. 1975, 110:677-684.

10.1001/archsurg.1975.01360110223039

7. Izbicki JR, Knoefel WT, Wilker DK, et al.: Accurate diagnosis of acute appendicitis: a retrospective and prospective analysis of 686 patients. Eur J Surg. 1992, 158:227-231.

8. Boleslawski E, Panis Y, Benoist S, et al.: Plain abdominal radiography as a routine procedure for acute abdominal pain of the right lower quadrant: prospective evaluation. World J Surg. 1999, 23:262-264. 10.1007/PL00013181

9. Puylaert JB: Imaging and intervention in patients with acute right lower quadrant disease . 
Baillieres Clin Gastroenterol. 1995, 9:37-51.

10. Torbati SS, Guss DA: Impact of helical computed tomography on the outcomes of emergency department patients with suspected appendicitis. Acad Emerg Med. 2003, 10:823-829.

11. Rosen MP, Sands DZ, Longmaid HE, et al.: Impact of abdominal CT on the management of patients presenting to the emergency department with acute abdominal pain. AJR Am J Roentgenol. 2000, 174:1391-1396. 10.2214/ajr.174.5.1741391

12. Balthazar EJ, Megibow AJ, Siegel SE, et al.: Appendicitis: prospective evaluation with highresolution CT. Radiology. 1991, 180:21-24. 10.1148/radiology.180.1.2052696

13. Siewert B, Raptopoulos B, Mueller MF, et al.: Impact of CT on diagnosis and management of acute abdomen in patients initially treated without surgery. AJR Am J Roentgenol. 1997, 168:173-178. 10.2214/ajr.168.1.8976942

14. Gorter RR, Eker HH, Gorter-Stam MA, et al.: Diagnosis and management of acute appendicitis. EAES consensus development conference 2015. Surg Endosc. 2016, 30:46684690. 10.1007/s00464-016-5245-7

15. Albano MC, Ross GW, Ditchek JJ, et al.: Resident interpretation of emergency CT scans in the evaluation of acute appendicitis. Acad Radiol. 2001, 8:915-918. 10.1016/S10766332(03)80772-9

16. Wise SW, Labuski MR, Kasales CJ: Comparative assessment of CT and sonographic techniques for appendiceal imaging. AJR Am J Roentgenol. 2001, 176:933-941. 10.2214/ajr.176.4.1760933

17. Balthazar EJ, Birnbaum BA, Yee J, et al.: Acute appendicitis: CT and US correlation in 100 patients. Radiology. 1994, 190:31-35. 10.1148/radiology.190.1.8259423

18. Funaki B, Grosskreutz SR, Funaki CN: Using unenhanced helical CT with enteric contrast material for suspected appendicitis in patients treated at a community hospital. Am J Roentgenol. 1998, 171:997-1001. 10.2214/ajr.171.4.9762983

19. Rao PM, Rhea JT, Novelline RA, et al.: Helical CT technique for the diagnosis of appendicitis: prospective evaluation of a focused appendix CT examination. Radiology. 1997, 202:139-144. 10.1148/radiology.202.1.8988203

20. Duddy MJ, Manns RA, Wormald SA: Injection rate: a factor in contrast reactions? . Clin Radiol. 1990, 41:42-43. 10.1016/S0009-9260(05)80931-6

21. Bernadino ME, Fishman EK, Jeffrey RB, et al.: Comparison of iohexol 300 and diatrizoate meglumine 60 for body CT: image quality, adverse reactions, and aborted/repeated examinations. Am J Roentgenol. 1992, 158:665-667. 10.2214/ajr.158.3.1739017

22. Rao PM, Rhea JT, Novelline RA, et al.: Helical CT combined with contrast material administered only through the colon for imaging of suspected appendicitis. Am J Roentgenol. 1997, 169:1275-1280. 10.2214/ajr.169.5.9353441

23. Lane MJ, Liu DM, Huynh MD, et al.: Suspected acute appendicitis: nonenhanced helical CT in 300 consecutive patients. Radiology. 1999, 213:341-346. 10.1148/radiology.213.2.r99nv44341

24. Hahn HB, Hoepner FU, Kalle T: Sonography of acute appendicitis in children: 7 years experience. Pediatr Radiol. 1998, 28:147-151. 10.1007/s002470050316

25. Pereira JM, Sirlin CB, Pinto PS, et al.: Disproportionate fat stranding: a helpful CT sign in patients with acute abdominal pain. RadioGraphics. 2004, 24:703-715. 10.1148/rg.243035084

26. Rao PM, Rhea JT, Novelline RA: Sensitivity and specificity of the individual CT signs of appendicitis: experience with 200 helical appendiceal CT examinations. J Comput Assist Tomogr. 1997, 21:686-692. 10.1097/00004728-199709000-00002

27. Pickhardt PJ, Levy AD, Rohrmann CA Jr, et al.: Primary neoplasms of the appendix manifesting as acute appendicitis: CT findings with pathologic comparison. Radiology. 2002, 224:775-781. 10.1148/radiol.2243011545

28. Rutledge RH, Alexender JW: Primary appendeceal malignancies: rare but important . Surgery. 1992, 111:244-250.

29. Rao PM, Rhea JT, Rattner DW, et al.: Introduction of appendiceal CT: impact on negative appendectomy and appendiceal perforation rates. Ann Surg. 1999, 229:344-349. 